\title{
Hölder continuity of harmonic quasiconformal mappings
}

Miloš Arsenović ${ }^{*}$, Vesna Manojlović ${ }^{2}$ and Matti Vuorinen ${ }^{3}$

* Correspondence: arsenovic@matf. bg.ac.rs

${ }^{1}$ Department of Mathematics, University of Belgrade, Studentski Trg 16, 11000 Belgrade, Serbia Full list of author information is available at the end of the article

\section{Abstract}

We prove that for harmonic quasiconformal mappings $\alpha$-Hölder continuity on the boundary implies $\alpha$-Hölder continuity of the map itself. Our result holds for the class of uniformly perfect bounded domains, in fact we can allow that a portion of the boundary is thin in the sense of capacity. The problem for general bounded domains remains open.

MSC 2010: 30C65.

Keywords: quasi-conformal maps, harmonic mappings, H?ö?lder continuity.

\section{Introduction}

The following theorem is the main result in [1].

Theorem 1.1. Let $D$ be a bounded domain in $\mathbb{R}^{n}$ and let $f$ be a continuous mapping of $\bar{D}$ into $\mathbb{R}^{n}$ which is quasiconformal in $D$. Suppose that, for some $M>0$ and $0<\alpha \leq 1$,

$$
|f(x)-f(y)| \leq M|x-y|^{\alpha}
$$

whenever $x$ and $y$ lie on $\partial D$. Then

$$
|f(x)-f(y)| \leq M^{\prime}|x-y|^{\beta}
$$

for all $x$ and $y$ on $\bar{D}$, where $\beta=\min \left(\alpha, K_{I}^{1 /(1-n)}\right)$ and $M^{\prime}$ depends only on $M, \alpha, n, K$ (f) and $\operatorname{diam}(D)$.

The exponent $\beta$ is the best possible, as the example of a radial quasiconformal map $f$ $(x)=|x|^{\alpha-1} x, 0<\alpha<1$, of $\overline{\mathbb{B}^{n}}$ onto itself shows (see [2], p. 49). Also, the assumption of boundedness is essential. Indeed, one can consider $g(x)=|x|^{a} x,|x| \geq 1$ where $a>0$. Then, $g$ is quasiconformal in $D=\mathbb{R}^{n} \backslash \overline{\mathbb{B}^{n}}$ (see [2], p. 49), it is identity on $\partial D$ and hence, Lipschitz continuous on $\partial D$. However, $\left|g\left(t e_{1}\right)-g\left(e_{1}\right)\right| \asymp t^{a+1}, t \rightarrow \infty$, and therefore, $g$ is not globally Lipschitz continuous on $D$.

This paper deals with the following question, suggested by P. Koskela: is it possible to replace $\beta$ with $\alpha$ if we assume, in addition to quasiconformality, that $f$ is harmonic? In the special case $D=\mathbb{B}^{n}$ this was proved, for arbitrary moduli of continuity $\omega(\delta)$, in [3]. Our main result is that the answer is positive, if $\partial D$ is a uniformly perfect set [4]. In fact, we prove a more general result, including domains having a thin, in the sense of capacity, portion of the boundary. However, this generality is in a sense illusory, because any harmonic and quasiconformal (briefly hqc) mapping extends harmonically

(c) 2011 Arsenovićć et al; licensee Springer. This is an Open Access article distributed under the terms of the Creative Commons Attribution License (http://creativecommons.org/licenses/by/2.0), which permits unrestricted use, distribution, and reproduction in any medium, provided the original work is properly cited. 
and quasiconformally across such portion of the boundary. Nevertheless, it leads to a natural open question: is the answer positive for arbitrary bounded domain in $\mathbb{R}^{n}$ ?

In the case of smooth boundaries much better regularity up to the boundary can be deduced, see [5]; related results for harmonic functions were obtained by Aikawa [6] and Sugawa [7].

We denote by $B(x, r)$ and $S(x, r)$ the open ball, respectively sphere, in $\mathbb{R}^{n}$ with center $x$ and radius $r>0$. We adopt the basic notation, terminology and definitions related to quasiconformal maps from [2]. A condenser is a pair $(K, U)$, where $K$ is a non-empty compact subset of an open set $U \subset \mathbb{R}^{n}$. The capacity of the condenser $(K, U)$ is defined as

$$
\operatorname{cap}(K, U)=\inf \int_{\mathbb{R}^{n}}|\nabla u|^{n} \mathrm{~d} V
$$

where infimum is taken over all continuous real-valued $u \in \mathrm{ACL}^{n}\left(\mathbb{R}^{n}\right)$ such that $u(x)$ $=1$ for $x \in K$ and $u(x)=0$ for $x \in \mathbb{R}^{n} \backslash U$. In fact, one can replace the $\mathrm{ACL}^{n}$ condition with Lipschitz continuity in this definition. We note that, for a compact $K \subset \mathbb{R}^{n}$ and open bounded sets $U_{1}$ and $U_{2}$ containing $K$ we have: $\operatorname{cap}\left(K, U_{1}\right)=0$ iff $\operatorname{cap}\left(K, U_{2}\right)=0$, therefore, the notion of a compact set of zero capacity is well defined (see [8], Remarks 7.13) and we can write $\operatorname{cap}(K)=0$ in this situation. For the notion of the modulus $M$ $(\Gamma)$ of a family $\Gamma$ of curves in $\mathbb{R}^{n}$ we refer to Väisälä [2] and Vuorinen [8]. These two notions are related: by results of Hesse [9] and Ziemer [10] we have

$$
\operatorname{cap}(K, U)=M(\Delta(K, \partial U ; U)),
$$

where $\Delta(E, F ; G)$ denotes the family of curves connecting $E$ to $F$ within $G$, see [2] or [8] for details.

In addition to this notion of capacity, related to quasiconformal mappings, we need Wiener capacity, related to harmonic functions. For a compact $K \subset \mathbb{R}^{n}, n \geq 3$, it is defined by

$$
\operatorname{cap}_{W}(K)=\inf \int_{\mathbb{R}^{n}}|\nabla u|^{2} \mathrm{~d} V,
$$

where infimum is taken over all Lipschitz continuous compactly supported functions $u$ on $\mathbb{R}^{n}$ such that $u=1$ on $K$. Let us note that every compact $K \subset \mathbb{R}^{n}$ which has capacity zero has Wiener capacity zero. Indeed, choose an open ball $B_{R}=B(0, R) \supset K$. Since $n \geq 2$ we have, by Hölder inequality,

$$
\int_{\mathbb{R}^{n}}|\nabla u|^{2} \mathrm{~d} V \leq\left|B_{R}\right|^{1-2 / n}\left(\int_{\mathbb{R}^{n}}|\nabla u|^{n} \mathrm{~d} V\right)^{2 / n}
$$

for any Lipschitz continuous $u$ vanishing outside $U$, our claim follows immediately from definitions.

A compact set $K \subset \mathbb{R}^{n}$, consisting of at least two points, is $\alpha$-uniformly perfect ( $\alpha>$ 0 ) if there is no ring $R$ separating $K$ (i.e. such that both components of $\mathbb{R}^{n} \backslash R$ intersect $K)$ such that $\bmod (R)>\alpha$, for definition of the modulus of a ring see [8]. We say that a compact $K \subset \mathbb{R}^{n}$ is uniformly perfect if it is $\alpha$-uniformly perfect for some $\alpha>0$.

We denote the $\alpha$-dimensional Hausdorff measure of a set $F \subset \mathbb{R}^{n}$ by $\Lambda_{\alpha}(F)$. 


\section{The main result}

In this section $D$ denotes a bounded domain in $\mathbb{R}^{n}, n \geq 3$. Let

$$
\Gamma_{0}=\{x \in \partial D: \operatorname{cap}(\bar{B}(x, \varepsilon) \cap \partial D)=0 \text { for some } \varepsilon>0\},
$$

and $\Gamma_{1}=\partial D \backslash \Gamma_{0}$. Using this notation we can state our main result.

Theorem 2.1. Assume $f: \bar{D} \rightarrow \mathbb{R}^{n}$ is continuous on $\bar{D}$, harmonic and quasiconformal in D. Assume $f$ is Hölder continuous with exponent $\alpha, 0<\alpha \leq 1$, on $\partial D$ and $\Gamma_{1}$ is uniformly perfect. Then $f$ is Hölder continuous with exponent $\alpha$ on $\bar{D}$.

If $\Gamma_{0}$ is empty we obtain the following

Corollary 2.2. If $f: \bar{D} \rightarrow \mathbb{R}^{n}$ is continuous on $\bar{D}$, Hölder continuous with exponent $\alpha$, $0<\alpha \leq 1$, on $\partial D$, harmonic and quasiconformal in $D$ and if $\partial D$ is uniformly perfect, then $f$ is Hölder continuous with exponent $\alpha$ on $\bar{D}$.

The first step in proving Theorem 2.1 is reduction to the case $\Gamma_{0}=\varnothing$. In fact, we show that existence of a hqc extension of $\mathrm{f}$ across $\Gamma_{0}$ follows from well known results. Let $D^{\prime}=D \cup \Gamma_{0}$. Then $D^{\prime}$ is an open set in $\mathbb{R}^{n}, \Gamma_{0}$ is a closed subset of $D^{\prime}$ and $\partial D^{\prime}=\Gamma_{1}$.

Clearly $\operatorname{cap}\left(K \cap \Gamma_{0}\right)=0$ for each compact $K \subset D^{\prime}$, and therefore, by Lemma 7.14 in [8], $\Lambda_{\alpha}\left(K \cap \Gamma_{0}\right)=0$ for each $\alpha>0$. In particular, $\Gamma_{0}$ has $\sigma$-finite $(n-1)$-dimensional Hausdorff measure. Since it is closed in $D^{\prime}$, we can apply Theorem 35.1 in [2] to conclude that $f$ has a quasiconformal extension $F$ across $\Gamma_{0}$ which has the same quasiconformality constant as $f$.

Since $\Gamma_{0}$ is a countable union of compact subsets $K_{j}$ of capacity zero and hence of Wiener capacity zero we conclude that $\Gamma_{0}$ has Wiener capacity zero. Hence, by a classical result (see [11]), there is a (unique) extension $G: \overline{D^{\prime}} \rightarrow \mathbb{R}^{n}$ of $f$ which is harmonic in $D^{\prime}$. Obviously, $F=G$ is a harmonic quasiconformal extension of $f$ to $\overline{D^{\prime}}$ which has the same quasiconformality constant as $f$.

In effect, we reduced the proof of Theorem 2.1 to the proof of Corollary 2.2. We begin the proof of Corollary 2.2 with the following

Lemma 2.3. Let $D \subset \mathbb{R}^{n}$ be a bounded domain with uniformly perfect boundary. There exists a constant $m>0$ such that for every $y \in D$ we have

$$
\operatorname{cap}\left(\bar{B}\left(y, \frac{d}{2}\right), D\right) \geq m, \quad d=\operatorname{dist}(y, \partial D) .
$$

Proof. Fix $y \in D$ as above and $z \in \partial D$ such that $|y-z|=d \equiv r$. Clearly $\operatorname{diam}(\partial D)=$ $\operatorname{diam}(D)>2 r$. Set $F_{1}=\bar{B}(z, r) \cap(\partial D)$ and $F_{2}=\bar{B}(z, r) \cap \bar{B}\left(\gamma, \frac{d}{2}\right), F_{3}=S(z, 2 r)$. Let $\Gamma_{i, j}=$ $\Delta\left(F_{i}, F_{j} ; \mathbb{R}^{n}\right)$ for $i, j=1,2$, 3. By Järvi and Vüorinen [4, Thm 4.1(3)], there exists a constant $a=a(E, n)>0$ such that

$$
M\left(\Gamma_{1,3}\right) \geq a
$$

while by standard estimates $[2,7.5]$ there exists $b=b(n)>0$ such that

$$
M\left(\Gamma_{2,3}\right) \geq b .
$$

Next, by Vuorinen $[8$, Cor 5.41] there exists $m=m(E, n)>0$ such that

$$
M\left(\Gamma_{1,2}\right) \geq m .
$$


Finally, with $B=\bar{B}(y, d / 2)$ we have

$$
\operatorname{cap}(B, D)=M\left(\Delta\left(B, \partial D ; \mathbb{R}^{n}\right)\right) \geq M\left(\Gamma_{1,2}\right) \geq m .
$$

In conclusion, from the above lemma, our assumption

$$
\left|f\left(x_{1}\right)-f\left(x_{2}\right)\right| \leq C\left|x_{1}-x_{2}\right|^{\alpha}, \quad x_{1}, x_{2} \in \partial D,
$$

and Lemma 8 in [1], we conclude that there is a constant $M$, depending on $m, n, K$ (f), $C$ and $\alpha$ only such that

$$
|f(x)-f(y)| \leq M|x-y|^{\alpha}, \quad y \in D, x \in \partial D, \quad \operatorname{dist}(y, \partial D)=|x-y| .
$$

However, an argument presented in [1] shows that the above estimate holds for $y \in$ $D, y \in \partial D$ without any further conditions, but with possibly different constant:

$$
|f(x)-f(y)| \leq M^{\prime}|x-y|^{\alpha}, \quad y \in D, x \in \partial D .
$$

The following lemma was proved in [12] for real valued functions, but the proof relies on the maximum principle which holds also for vector valued harmonic functions, hence lemma holds for harmonic mappings as well.

Lemma 2.6. Assume $h: \bar{D} \rightarrow \mathbb{R}^{n}$ is continuous on $\bar{D}$ and harmonic in $D$. Assume for each $x_{0} \in \partial D$ we have

$$
\sup _{B_{r}\left(x_{0}\right) \cap D^{\prime}}\left|h(x)-h\left(x_{0}\right)\right| \leq \omega(r) \quad \text { for } \quad 0<r \leq r_{0} .
$$

Then $|h(x)-h(y)| \leq \omega(|x-y|)$, whenever $x, y \in D$ and $|x-y| \leq r_{0}$.

Now we combine (2.5) and the above lemma, with $r_{0}=\operatorname{diam}(D)$, to complete the proof of Corollary 2.2 and therefore of Theorem 2.1 as well.

\section{Acknowledgements}

M. Arsenovic's work was supported by Ministry of Science, Serbia, project M144010, V. Manojlovic's work by Ministry of Science, Serbia, project M174024, and M. Vuorinen's work by the Academy of Finland, project 2600066611.

\section{Author details}

'Department of Mathematics, University of Belgrade, Studentski Trg 16, 11000 Belgrade, Serbia ${ }^{2}$ Faculty of Organizational Sciences, University of Belgrade, Jove llica 154, 11000 Belgrade, Serbia ${ }^{3}$ Department of Mathematics, University of Turku, 20014 Turku, Finland

\section{Authors' contributions}

VM conceived of the study and participated in its design and coordination, MV and MA proved the main result. All authors read and approved the final manuscript.

\section{Competing interests}

The authors declare that they have no competing interests.

Received: 29 December 2010 Accepted: 23 August 2011 Published: 23 August 2011

\section{References}

1. Martio, O, Näkki, R: Boundary Hölder continuity and quasiconformal mappings. J Lond Math Soc. 44(2), 339-350 (1991). doi:10.1112/jlms/s2-44.2.339

2. Väisälä, J: Lectures on n-dimensional Quasiconformal Mappings. Lecture Notes in Mathematics. 229, Springer, Berlin (1971)

3. Arsenović, $M$, Božin, $V$, Manojlović, $V$ : Moduli of continuity of harmonic quasiregular mappings in $\mathbb{B}^{n}$. Potential Anal. 34(3):283-291 (2011). doi:10.1007/s11118-010-9195-8

4. Järvi, P, Vuorinen, M: Uniformly perfect sets and quasiregular mappings. J Lond Math Soc. 54(2):515-529 (1996)

5. Kalaj, D: Quasiconformal and harmonic mappings between Jordan domains. Math Z. 260(2), 237-252 (2008). doi:10.1007/s00209-007-0270-9

6. Aikawa, H: Hölder continuity of the Dirichlet solution for a general domain. Bull Lond Math Soc. 34(6), 691-702 (2002). doi:10.1112/S0024609302001522

7. Sugawa, T: On boundary regularity of the Dirichlet problem for plane domains(preprint, 1999). 
8. Vuorinen, M: Conformal Geometry and Quasiregular Mappings. Lecture Notes in Mathematics. 1319, Springer, Berlin (1988)

9. Hesse, J: A p-extremal length and p-capacity equality. Ark Mat. 13, 131-141 (1975). doi:10.1007/BF02386202

10. Ziemer, WP: Extremal length and p-capacity. Michigan Math J. 16, 43-51 (1969)

11. Carleson, L: Selected Problems on Exceptional Sets. Van Nostrand Mathematical Studies Princeton. (1967)

12. Caffarelli, LA, Kinderlehrer, D: Potential methods in variational inequalities. J Anal Math. 37, 285-295 (1980). doi:10.1007/ BF02797689

doi:10.1186/1029-242X-2011-37

Cite this article as: Arsenović et al: Hölder continuity of harmonic quasiconformal mappings. Journal of Inequalities and Applications 2011 2011:37.

\section{Submit your manuscript to a SpringerOpen ${ }^{\circ}$} journal and benefit from:

$\rightarrow$ Convenient online submission

- Rigorous peer review

- Immediate publication on acceptance

- Open access: articles freely available online

- High visibility within the field

- Retaining the copyright to your article

Submit your next manuscript at $\boldsymbol{\nabla}$ springeropen.com 